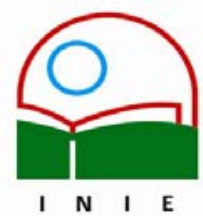

\title{
EL XSLT COMO HERRAMIENTA PARA EL DISEÑO DE SISTEMAS LEXICOGRÁFICOS INFORMATIZADOS: APLICACIÓN A ADJETIVOS DE UN LÉXICO META
}

\author{
Marta Rojas Porras ${ }^{1}$ \\ Antonio Leoni de León ${ }^{2}$
}

\begin{abstract}
Resumen: El artículo expone el procedimiento seguido para la clasificación y análisis de los adjetivos de un léxico meta propuesto para el Segundo Ciclo de la Educación General Básica. Se utiliza el XSLT como herramienta para el diseño de sistemas lexicográficos informatizados y se prueba su idoneidad, pues entre otras ventajas, éste favorece la manipulación del amplio corpus, por parte de quienes investigan y planifican su enseñanza y su incorporación a la competencia comunicativa de la niñez costarricense.
\end{abstract}

Palabras clave: LÉXICO META/ SISTEMAS LEXICOGRÁFICOS INFORMATIZADOS/ LINGÜÍSTICA APLICADA/ ENSEÑANZA DEL ESPAÑOL/ PLANIFICACIÓN LINGÜÍSTICA/

\begin{abstract}
The article exposes the procedure followed for the classification and analysis of the adjectives of a meta-vocabulary proposed for the Second Cycle of the Basic General Education. The XSLT is used as a tool for the design of computerized lexicographical systems; and its suitability is proven by, among other advantages, its ability to manipulate the vast corpus. This ability is given to those who investigate and plan the education of the vocabulary and its addition to the communication proficiency of the Costa Rica's childhood.
\end{abstract}

Key words: META-VOCABULARY/ COMPUTERIZED LEXICOGRAPHIC SYSTEMS/ APPLIED LINGUISTICS/ TEACHING OF SPANISH/ LINGUISTIC PLANNING/

\section{Introducción}

Los datos que se presentan en las siguientes líneas se derivan de la investigación Desarrollo de la madurez léxica: propuesta metodológica para sexto año de la Educación General Básica, llevada a cabo en el Instituto de Investigación en Educación (INIE) por la profesora Marta Rojas y en la que participa, como investigador asociado, el Dr. Víctor Manuel Sánchez Corrales.

\footnotetext{
${ }^{1}$ Licenciada en Filología Española de la Universidad de Costa Rica, Profesora catedrática en Escuela de Filología, Lingüística y Literatura e investigadora en el Instituto de Investigación en Educación ambos de la Universidad de Costa Rica. Directora de la Revista Educación de la Universidad de Costa Rica. Correo electrónico: merojasporras@yahoo.com

${ }^{2}$ Master en Lingüística, Licenciado en Filología Española ambos títulos de la Universidad de Costa Rica. Se encuentra realizando estudios de doctorado en Lingüística Informática en la Universidad de Ginebra, Suiza. Profesor universitario del curso de Esperanto. Correo electrónico: tiberino@yahoo.com
}

Artículo recibido: 8 de marzo, 2005

Aprobado: 25 de abril, 2005 
El propósito de este estudio es proponer un instrumental teórico y práctico para mejorar la experiencia de la enseñanza y del aprendizaje del léxico en la educación formal costarricense.

Como apunta Gómez Molina (1996), la finalidad de la enseñanza del léxico es que las unidades léxicas pasen a la competencia comunicativa del individuo, dado el saber del vocabulario como elemento estructurador del pensamiento -función simbólica-y su necesidad para la interacción social -función comunicativa-. Es evidente que el léxico es el componente lingüístico que mejor traba el conocimiento del mundo y la apertura comunicativa del hablante porque el lenguaje es un medio para conseguir objetivos y para simbolizar relaciones.

No obstante, según lo plantea Araya (2001), en Costa Rica los programas de Español del Tercer Ciclo (y presumiblemente, según los autores de este artículo, los de los demás niveles) se caracterizan por una limitación cuantitativa en el campo de la enseñanza del léxico. Hasta 1990 se evidencia la falta de un marco metodológico, lo cual se expresa en una incorporación confusa de este conocimiento dentro de otros campos de estudio de la lengua, esto es, el léxico se subordina sensiblemente al resto de los componentes. Anota esta investigadora que en 1991 se introduce la noción de campos semánticos en actividades complementarias al plan de estudios, y además se ofrecen conocimientos morfológicos muy completos. Observa también que el programa vigente a partir de 1995 y hasta ahora, a pesar de que destina solo un $9 \%$ a la enseñanza del léxico, es el único que ofrece en su preámbulo una definición precisa del léxico, y menciona la metodología por utilizar en su desarrollo. Amplía asimismo la noción de variedad léxica y semántica, y estudia el lenguaje de los adolescentes y el español de Costa Rica. Por último, en las guías didácticas se detallan la metodología y las actividades, y se considera la competencia lingüística de los educandos. Aún así, a pesar de estos avances el estudio del componente léxico no se aborda a profundidad.

También es necesario aclarar que los estudios sobre la enseñanza del léxico en español como lengua materna son casi inexistentes, y que los pocos que se encuentran son aplicados a la enseñanza del español para extranjeros. 


\section{El léxico meta}

Ante el reto de elaboración de este instrumental teórico y práctico, había que dar respuesta a la pregunta “¿Qué vocabulario enseñar?", para posteriormente plantear el cómo. A este corpus del vocabulario por enseñar es al que se ha denominado "léxico meta".

El léxico meta se determinó a partir de tres inventarios:

1. Léxico básico escolar costarricense: diccionario de frecuencias: Corpus que corresponde a una muestra nacional de textos escritos por niños de escuelas urbanas y rurales de las 18 regiones educativas del país. Investigación adscrita a la Escuela de Filología, Lingüística y Literatura, en colaboración con el IIMEC y realizada por Marta Rojas Porras.

2. Léxico básico escolar costarricense: Investigación del programa ELEHXICÓS, a cargo del Dr. Víctor Manuel Sánchez y la Dra. Marielos Murillo Rojas, quienes trabajaron una muestra nacional, tanto de la oralidad como de la escritura.

3. El léxico de los libros de texto de sexto año de Ciencias, Matemáticas, Estudios Sociales y Español, de la Serie Hacia el Siglo XXI, el cual, una vez sometido a los procesos del filtro, constituirá el léxico meta. Este corpus se elaboró en conjunto IIMEC-ELEXHICÓS.

Para determinar el léxico meta, mediante un programa diseñado por el M.L. Antonio Leoni, el vocabulario de los libros fue cotejado con los dos corpus de léxicos básicos anteriormente señalados, de manera que las palabras que formaban parte del uso (léxicos básicos) no debían aparecer en el léxico meta. De esta forma el uso (léxicos básicos) fue filtro para la definición del léxico meta.

En resumen, el léxico meta quedó constituido por 445 lexemas (sustantivos, adjetivos, adverbios y verbos), de los cuales 125 corresponden a adjetivos, categoría a la que se refiere el trabajo presentado. De esos adjetivos, para efectos de este artículo, se seleccionaron 52 . 


\section{Tratamiento metodológico para organizar los adjetivos del corpus}

Para elaborar las actividades y ejercicios con miras a una propuesta metodológica significativa, en la que el estudiante incorpore sus conocimientos a través del ludo (aprender jugando), de las habilidades de pensamiento y del desarrollo de valores, se hizo necesaria una organización del material que permitiera su posterior manipulación o manejo.

Debe entenderse que aunque en esta presentación los adjetivos, sus sinónimos y antónimos aparecen como palabras aisladas, en la propuesta metodológica estos serán incorporados a contextos relacionados con la experiencia de los estudiantes y en un marco de asociación semántica por oposición, por semejanza, por relaciones de proximidad (parte y todo, general-particular, causa-efecto), así como con la consulta adecuada y creativa al diccionario.

De esta manera resultó conveniente una primera aproximación al corpus, ya en asocio con el M.L. Leoni; etapa del trabajo que apenas está en proceso inicial; pero de la cual nos pareció oportuno mostrar las posibilidades. El documento que se genera de esta aplicación, así como los datos que se presentan en este artículo corresponden a material de uso exclusivo de los investigadores. A partir de ellos se elaborarán las actividades y ejercicios de la propuesta metodológica.

En esta primera etapa, los adjetivos se clasificaron en los siguientes rubros:

1. En cuanto a lo sintáctico:

Se analiza el orden del adjetivo (pospuesto o antepuesto al sustantivo, o ambas) y sus comportamientos como atributo (permanente, contingente o con sujeto oracional).

2. En cuanto a lo semántico:

Para cada adjetivo se establecieron relaciones de sinonimia y antonimia y se determinaron los sinónimos del antónimo. La mayoría de los sinónimos y antónimos no son extraídos del corpus meta, sino aportados por los investigadores. Posteriormente estas listas deberán someterse a juicio de expertos para determinar los adjetivos adecuados para sexto año.

En la página siguiente, unos ejemplos de esta clasificación: 
TABLA 1

Muestra de la clasificación del corpus

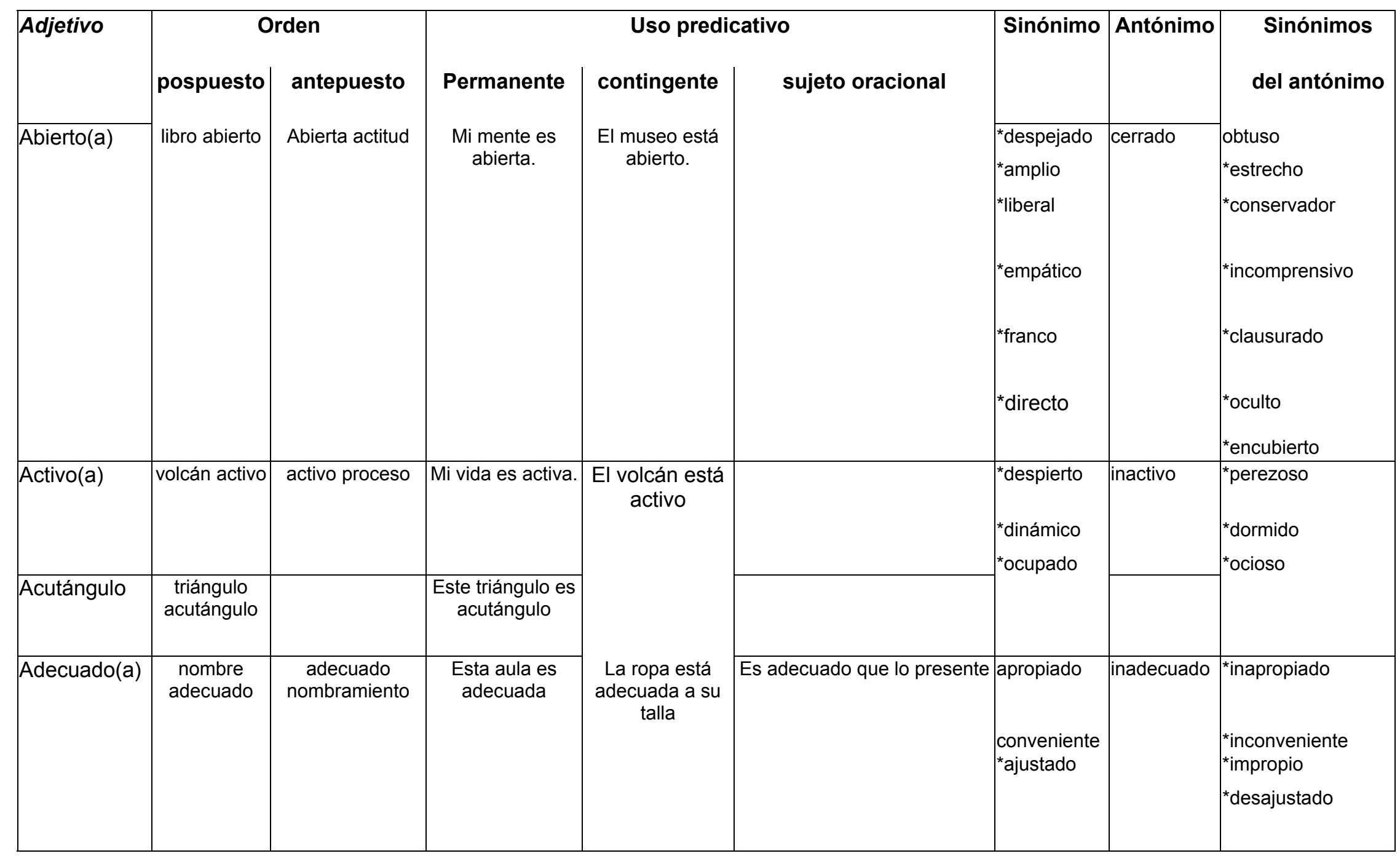




\section{- Los adjetivos y su representación}

La estructura del léxico y su representación formal tienen una importancia particular en la lingüística aplicada, por cuanto es factible crear sistemas automatizados que faciliten el tratamiento de los lemas con diversos propósitos prácticos, de los cuales el ámbito educativo es, quizás, el más relevante. Un proyecto de gran interés es WordNet (Miller et al., 1990), defininido a grandes rasgos como una base de datos léxico-conceptual, inspirada en teorías psicolingüísticas contemporáneas sobre la memoria léxica humana, y que constituye un sistema relacional del conocimiento.

El concepto de WordNet ha sido fértil: existen versiones en varios idiomas (entre ellos el español), y se encuentra incluso una propuesta universalista que permite traducir sentidos entre wordnets de distintas lenguas.

\section{- Los adjetivos en WordNet}

En nuestro caso particular, se busca aplicar al español algunos principios básicos de WordNet relacionados con la organización de los adjetivos y que pueden resultar útiles en la creación de bases de datos léxicas. En WordNet la clasificación general de los adjetivos es la siguiente:

i. Descriptivos: le atribuyen un valor a un sustantivo. Un adjetivo es descriptivo si cumple con la siguiente fórmula: $A(x)=A d j$, la cual en la práctica se expresa como en (1):

(1) PESO (paquete) = liviano

La organización de estos adjetivos se basa principalmente en la antonimia. En muchas ocasiones, no existe un antónimo directo para un término dado, pero sí hay un grupo de términos asociados con uno que sí posee un antónimo; tal relación se denomina antonimia indirecta. Posee la forma de un grupo de adjetivos asociados a un término adjetivo principal (es decir, que sí posee antónimo), los cuales son antónimos indirectos del término o los términos asociados con su antónimo (Miller et al., 1990, p. 29).

ii. Modificadores de referencia: Es una clase cerrada de adjetivos que pueden hacer variar la interpretación del sintagma nominal; por ejemplo, en (2)
a. Mi viejo amigo
b. Mi nuevo amigo 
el sustantivo amigo se encuentra modificado por las características semánticas de los sintagmas adjetivos nuevo y viejo. En WordNet los adjetivos modificadores de referencia llevan, en su mayoría, marcas de realización para indicar que estos se manifiestan como atributos y no como predicados (que siempre se encuentran dentro del sintagma nominal).

iii. Relacionales: Estos, paralelamente a los adjetivos modificadores de referencia, se manifiestan, por lo general, como atributos y no como predicados. Es el caso de adjetivos como dental:

a. La higiene dental es necesaria

b. $\quad *$ La higiene es dental

Estos adjetivos a menudo se derivan de sustantivos, no presentan gradación ni tampoco antónimos directos, por lo que no pueden incorporarse a los grupos que caracterizan a los adjetivos descriptivos. En WordNet los relacionales tienen un puntero que los vincula con los sustantivos correspondientes.

En el marcaje de los adjetivos, la base de datos cuenta con las marcas siguientes de restricción sintáctica:

(p) = sólo se realizan como predicado.

(a) = prenominales. Siempre anteceden al sustantivo que modifican.

(ip) = postnominales. Siempre suceden al sustantivo que modifican.

Conviene aclarar que la presente investigación aún no ha comenzado su análisis de los sustantivos, por lo que el etiquetado relacional queda, por el momento, pendiente.

Ahora bien, la clasificación de adjetivos se lleva a cabo manualmente, para lo cual se elabora una tabla con siete columnas, cada una de las cuales incluye los valores siguientes:

1. Lema

2. Posición del adjetivo con respecto al sustantivo (pospuesto o antepuesto)

3. Uso del adjetivo como atributo en oraciones copulativas

4. Sinonimia 


\section{Antonimia}

6. Antonimia indirecta (sinónimos de los antónimos)

7. Clasificación.

Sin embargo, dada la dificultad de manejo de este formato, se impone su transformación en una estructura más manipulable. Es aquí donde se recurre al Lenguaje de etiquetado extensible (XML por sus siglas en inglés): un descriptor y estructurador de contenidos multilingües para intercambio de información entre computadoras o entre seres humanos.

\section{- Lenguaje etiquetado de adjetivos (LDA)}

Todo documento XML es representable como un árbol, por lo que es lícito decir que uno de los investigadores, el M.L. Leoni, creó un documento XML, al que llamó AdjML (ver Anexo $\mathrm{C}$ ), en el cual cada rama es el esqueleto de un artículo lexicográfico que contiene un adjetivo del corpus, y cada hoja corresponde a una de las últimas seis columnas de la tabla arriba descrita. $^{2}$

Para transformar la tabla en un documento XML, hubo que convertirla primero en un documento Excel, convertido a su vez en un documento de valores separados por comas (CSV), transformado en $\mathrm{Perl}^{3}$ mediante un programa (ver Anexo A) que también se escribió y que convierte el archivo CSV en un documento XML, más fácil de manejar.

Una vez hecho esto, por medio de un programa escrito en el Lenguaje de transformaciones de etiquetado extensible de hoja de estilo (XSLT; ver Anexo B), se transformó el documento XML en uno que cumpliera los parámetros de LDA.

Una vez obtenido el documento en LDA, el cual describe ciertas características de los adjetivos, y puede utilizarse en diversas operaciones de consulta, es posible comenzar a tratar los contenidos mismos.

\footnotetext{
${ }^{2}$ Son seis en vez de siete porque la primera columna corresponde a los lemas, los cuales serán el encabezado de nuestro documento AdjML.

${ }^{3}$ El Lenguaje práctico de extracción y reporte (Perl, por sus siglas en inglés) es una herramienta poderosa para manejar grandes cantidades de información textual.
} 
XSLT es un lenguaje apto para navegar por cualquier árbol de un documento XML, de forma que se puede extraer información de él y, además, a partir de los datos obtenidos, ${ }^{4}$ producir otros documentos en diversos formatos. Ello permite intercambiar información fácil y confiablemente; además, posibilita actualizarla a partir de otros que cumplan con las normas del XML. Para ilustrar estos propósitos, se ha escrito un documento en XSLT, llamado AdjML->HTML: antonimia (ver Anexo D), que extrae la información sobre la antonimia de AdjML y la convierte en un documento HTML.

LDA, por las características de todo documento en XML, puede interactuar con otros documentos XML y, más importante aún, con otras herramientas de tratamiento de documentos en XML, por lo que hace posible la interacción a través de cualquier red informática. Esto lleva a pensar en las posibles aplicaciones, como una base de datos interinstitucional (e incluso internacional) que comparta información sobre sus áreas de competencia e investigación; también sería posible crear bases de datos de fácil consulta o juegos interactivos de aprendizaje. Todo esto sin que los usuarios tengan que darse cuenta de los detalles y las sutilezas tecnológicas que subyacen a esos sistemas.

Los adjetivos en LDA se organizan como sigue:

1. Lema

2. Posposición

3. Anteposición

4. Rasgos del verbo auxiliar:
a. Permanente
b. Contingente

5. Los adjetivos en LDA

6. Con sujeto oracional (adjetivos referidos a expletivos)

7. Sinonimia

8. Antonimia

9. Antonimia indirecta

10. Antosinónimos.

\footnotetext{
${ }^{4}$ Es lo que algunos llaman la separación del contenido y su representación. En XML los contenidos están descritos por una convención que el investigador establece; XSLT permite que esos contenidos estén accesibles en otros formatos como PDF, HTML o, incluso, XML.
} 
Los ejemplos de esta clasificación se encuentran en la Tabla 1.

Una muestra del documento base de valores separados por comas (CSV) se ofrece en el Anexo A; el programa de conversión de CSV a XML, en el Anexo B; el programa en XSLT que convierte al documento XML en LDA, en el Anexo F; y una representación parcial del LDA, en el Anexo C. Los programas de extracción de la información de XSLT figuran en los Anexos D, G y H junto con los resultados respectivos.

\section{Resultados}

1. Comportamiento sintáctico de los adjetivos de la muestra del léxico meta

1. 1. En cuanto al orden respecto del núcleo (sustantivo) en la frase nominal

- Adjetivos exclusivamente antepuestos: Ninguno.

- Adjetivos exclusivamente pospuestos:

1. acutángulo

2. ambiental

3. antártico(a)

4. ártico(a)

5. atlántico(a)

6. atómico(a)

7. binario(a)

8. biológico(a)

9. calórico(a)

10. capilar

11. circulatorio(a)

12. colonial

13. derecho(a) (I)

14. digital

15. editorial

16. eléctrico(a)

17. encargado(a) (I y II) 

18. eólico(a)
19. escrito(a)
20. fotográfico(a)
21. intermediario(a)
22. interplanetario(a)
23. metálico(a)
24. occidental
25. opuesto(a)
26. periodístico(a)
27. sexual

- Adjetivos cuya aparición puede ser pospuesta y antepuesta:

1. activo(a)

2. adecuado(a)

3. agudo(a)

4. alumbrado(a)

5. apasionante

6. apropiado(a)

7. astronómica(o)

8. benefactor(a)

9. boscoso(a)

10. carretero(a)

11. caudaloso(a)

12. cerrado(a)

13. cómodo(a)

14. cuadrado(a)

15. derecho(a)

16. destacado(a)

17. eficaz

18. evidente

19. fluido(a)

20. natural

21. original

22. pesado(a) 
23. probable

24. similar

\subsection{En cuanto al uso predicativo}

\section{- Con verbo copulativo + permanente ("ser"):}

La mayoría integra este tipo de estructura, exceptuando a los participiales: alumbrado(a) encargado(a) (I y II) y escrito(a $)^{5}$. Tampoco lo admiten atlántico(a) ni calórico(a).

- Con verbo copulativo + contingente ("estar"):

1. abierto(a)

2. $\operatorname{activo(a)}$

3. adecuado(a)

4. alumbrado(a)

5. apasionante

6. apropiado(a)

7. caudaloso(a)

8. cerrado(a)

9. cómodo(a)

10. cuadrado(a)

11. derecho(a) (ii)

12. destacado(a)

13. encargado(a) (i y ii)

14. escrito(a)

15. fluido(a)

16. opuesto(a)

17. original

18. pesado(a)

19. similar.

${ }^{5}$ En caso de construirse con ser, este verbo abandona su valor copulativo para tomar el de auxiliar, y el adjetivo también pierde su carácter de tal para asumir la carga verbal, de modo que se está ante una construcción pasiva. 
- Atributos de sujeto oracional (expletivo):

1. adecuado

2. apropiado

3. eficaz

4. evidente

5. original

6. probable

\section{Comportamiento semántico de los adjetivos de la muestra}

\subsection{Sinónimos}

La Tabla 2 muestra los sinónimos de los adjetivos del léxico meta:

\section{Tabla 2}

\begin{tabular}{|c|c|}
\hline Adjetivo & Sinónimos \\
\hline Abierto & $\begin{array}{c}\text { despejado, amplio, liberal, empático, franco, } \\
\text { directo }\end{array}$ \\
\hline Activo & despierto, dinámico, ocupado \\
\hline Adecuado & ${ }^{*}$ apropiado, ${ }^{*}$ conveniente, ajustado \\
\hline Agudo & fino, delgado, penetrante \\
\hline Alumbrado & iluminado, irradiado, enfocado \\
\hline Apasionante & arrebatador, estimulante, incitante, \\
& provocativo \\
\hline Apropiado & adecuado, propicio, oportuno, pertinente, \\
& correspondiente, apto \\
\hline Astronómico & enorme, inmenso, descomunal, mayúsculo \\
\hline Benefactor & filántropo, protector, caritativo \\
\hline Biológico & orgánico, viviente \\
\hline Boscoso & selvático, forestal, frondoso, nemoroso, \\
& arbolado \\
\hline
\end{tabular}




\begin{tabular}{|c|c|}
\hline Caudaloso & crecido, vasto, copioso, profundo \\
\hline Cerrado & $\begin{array}{l}\text { clausurado, bloqueado, obstruido, trancado, } \\
\text { impenetrable, tapado, hermético, sellado, } \\
\text { incomprensivo, obstinado, testarudo }\end{array}$ \\
\hline Circulatorio & arterial, venoso \\
\hline Colonial & ultramarino, subordinado \\
\hline Cómodo & $\begin{array}{l}\text { confortable, desahogado, relajado, } \\
\text { relajante, placentero, oportuno, } \\
{ }^{*} \text { conveniente }\end{array}$ \\
\hline Derecho & diestro \\
\hline Derecho & recto, erguido, firme, tieso \\
\hline Destacado & $\begin{array}{c}\text { resaltado, reforzado, enfatizado, acentuado, } \\
\text { señalado, sobresaliente, prominente, } \\
\text { eminente }\end{array}$ \\
\hline Eficaz & eficiente, fructífero, provechoso, potente \\
\hline Encargado & pedido, solicitado, encomendado \\
\hline Encargado & responsable, designado, encomendado \\
\hline Escrito & anotado, apuntado, impreso \\
\hline Evidente & patente, notorio \\
\hline Fluido & $\begin{array}{l}\text { chorreado, manado, segregado, secretado, } \\
\text { destilado, continuo, líquido, ágil }\end{array}$ \\
\hline Intermedio & central, equidistante, transicional \\
\hline Metálico & férreo, chapado, mineral \\
\hline Natural & espontáneo, originario \\
\hline Occidental & eurocéntrico \\
\hline Opuesto & $\begin{array}{c}\text { contrario, adverso, contrapuesto, } \\
\text { divergente, antitético, inverso, incompatible, } \\
\text { irreconciliable }\end{array}$ \\
\hline Original & $\begin{array}{c}\text { primigenio, prototípico, singular, peculiar, } \\
\text { novedoso, especial, excepcional, curioso, } \\
\text { extravagante, estrafalario }\end{array}$ \\
\hline Periodístico & Informativo \\
\hline Pesado & Difícil, dificultoso, trabajoso, recargado, \\
\hline
\end{tabular}




\begin{tabular}{|c|c|}
\hline & $\begin{array}{c}\text { gravoso, oneroso, indigestible, insoportable } \\
\text { Probable }\end{array}$ \\
\hline Sexual & $\begin{array}{r}\text { Demostrable, constatable, fidedigno, } \\
\text { creíble, presumible, presunto }\end{array}$ \\
\hline Similar & $\begin{array}{c}\text { Semejante, parecido, paralelo, análogo, } \\
\text { comparable, parangonable, emparentado, } \\
\text { cercano }\end{array}$ \\
\hline
\end{tabular}

Se anotan con asterisco* los adjetivos que se derivan del corpus

\section{- Adjetivos de la muestra sin sinónimo léxico:}

1. acutángulo

2. ambiental

3. antártico(a)

4. ártico(a)

5. atlántico(a)

6. atómico(a)

7. binario(a)

8. calórico(a)

9. capilar

10. carretero(a)

11. cuadrado(a)

12. digital

13. editorial

14. eléctrico(a)

15. eólico(a)

16. fotográfico(a)

17. interplanetario(a) 


\subsection{Antónimos}

Los resultados de la antonimia se presentan en la Tabla 3.

\section{Tabla 3}

\begin{tabular}{|c|c|c|}
\hline \multicolumn{3}{|r|}{ Antonimia } \\
\hline Lema & Antónimo directo & Sinónimo del anónimo \\
\hline abierto(a) & *cerrado & $\begin{array}{l}\text { *obtuso estrecho conservador incomprensivo } \\
\text { clausurado oculto encubierto }\end{array}$ \\
\hline Activo(a) & inactivo & perezoso dormido ocioso \\
\hline adecuado(a) & inadecuado & inapropiado inconveniente impropio desajustado \\
\hline Agudo(a) & grave & \\
\hline alumbrado(a) & penumbroso & oscurecido ensombrecido \\
\hline ambiental & genético & congénito innato constitucional hereditario \\
\hline antártico(a) & *ártico & \\
\hline apasionante & desapasionante & desmotivante desalentador aburrido frío \\
\hline apropiado(a) & inapropiado & impropio inoportuno \\
\hline Ártico(a) & *antártico & \\
\hline astronómica(o) & diminuto & ínfimo nimio minúsculo \\
\hline benefactor(a) & perjudicial & negligente desconsiderado destructor maléfico \\
\hline biológica(o) & muerto & inerte inorgánico mineral fósil \\
\hline boscoso(a) & deforestado & talado desértico \\
\hline caudaloso(a) & ${ }^{*} \operatorname{seco}$ & vacío decrecido \\
\hline cerrado(a) & *abierto & amplio despejado disponible accesible \\
\hline colonial & soberano & emancipado autónomo insubordinado \\
\hline
\end{tabular}




\begin{tabular}{|c|c|c|}
\hline cómodo(a) & incómodo & $\begin{array}{l}\text { embarazoso penoso dificultoso perturbador tenso } \\
\text { inoportuno inconveniente }\end{array}$ \\
\hline derecha(o) & izquierdo & zurdo \\
\hline derecho(a) & torcido & inclinado ladeado sesgado jorobado \\
\hline destacado(a) & debilitado & menguado anulado eclipsado deficiente mediocre \\
\hline Eficaz & ineficaz & $\begin{array}{l}\text { inoperante inútil inservible infructífero } \\
\text { insatisfactorio }\end{array}$ \\
\hline escrito(a) & oral & hablado \\
\hline evidente & oculto & latente secreto encubierto subrepticio \\
\hline Fluido(a) & contenido & $\begin{array}{l}\text { espeso detenido denso viscoso trabado atorado } \\
\text { atascado atajado }\end{array}$ \\
\hline intermedio(a) & extremo & lateral marginal inicial final \\
\hline natural & artificial & imitado forzado postizo sintético antropogénico \\
\hline occidental & oriental & exótico \\
\hline opuesto(a) & igual & $\begin{array}{l}\text { traslapado relacionado convergente afín } \\
\text { compatible }\end{array}$ \\
\hline original & repetido & copiado duplicado plagiado pirateado \\
\hline pesado(a) & liviano & ligero leve tenue suave fácil \\
\hline probable & improbable & indemostrable increíble inexistente \\
\hline sexual & asexual & ascético virginal casto \\
\hline similar & disímil & $\begin{array}{l}\text { diferente distinto disparejo desigual *opuesto } \\
\text { lejano }\end{array}$ \\
\hline
\end{tabular}

Los lemas marcados con asterisco no pertenecen al corpus. 
- Adjetivos sin antónimo

1. acutángulo

2. atlántico(a)

3. atómico(a)

4. binario

5. calórico(a)

6. capilar

7. carretero(a)

8. cuadrado(a)

9. digital

10. editorial

11. eléctrico(a)

12. encargado(a)(1y2)

13. eólico(a)

14. fotográfico(a)

15. interplanetario(a)

16. metálico(a)

17. periodístico(a)

\section{Conclusiones}

XSLT es una herramienta de gran valor para el diseño de sistemas lexicográficos informatizados, puesto que:

- El lexicógrafo tiene el control sobre el flujo de la información en el sistema.

- La arquitectura es clara y fácil de comprender.

- Permite avanzar rápidamente en la investigación y luego un informático puede comprender sin problemas las ideas expresadas en XML/XSLT.

- Es posible utilizar de manera inmediata la información en redes informáticas (internet, por ejemplo). 
- Favorece la manipulación del corpus, de manera que este pueda ser utilizado por los investigadores y, para el caso particular, su organización proporciona pistas para el tratamiento didáctico y lúdico del mismo, proceso para el cual los datos así presentados representan un insumo.

- La posibilidad de analizar tanto aspectos sintácticos como semánticos proporciona información valiosa para la elaboración de las actividades que incorporen el léxico meta en contextos textuales y pragmáticos.

- Para el caso que nos ocupa, el XSLT resulta una herramienta de gran utilidad, pues la organización semántica y morfosintáctica que proporciona pistas para el tratamiento didáctico del léxico meta propuesto para la enseñanza.

- La información obtenida de la aplicación del XSLT, puesta en manos de maestros y maestras, de quienes atienden las asesorías de español y de los planificadores/as lingüísticos/as, muestra relaciones morfosintácticas y semánticas que podrían favorecer las propuestas del tratamiento del contenido léxico, tanto en el nivel del aula como en el microsistema, según la condición.

\section{Referencias}

Araya, Kattia. (2001). La enseñanza del léxico en los programas de estudio de Español del Tercer Ciclo (1971-1995). Káñina, Revista de Artes y Letras de la Universidad de Costa Rica. XXV (2): 37-59.

Bray, Tim; et al. (2003). Espacios de nombre en XML. Recuperado el 12 de junio del 2003, de http://html.conclase.net/w3c/xml-names-es/.

. (2003). Extensible Markup Language (XML) 1.0. Recuperado el 12 de junio del 2003, de http://www.w3.org/TR/REC-xml/

. (2003). Extensible Markup Language (XML) 1.0 - El lenguaje extensible de marcas (XML) 1.0. Recuperado el 12 de junio del 2003, de http://www.sidar.org/recur/desdi/traduc/es/xml/xml1/index.html.

Clark, James; de Rose, Steve. (2001). Lenguaje de caminos XML (XPath). Recuperado el 19 de octubre del 2001, de http://www.sidar.org/recur/desdi/traduc/es/xml/xpath.html. 
Fellbaum, Christiane. (1998). WordNet: An Electronic Lexical Database. Cambridge, Massachusetts: MIT Press.

Gómez Molina, Juan Ramón. (1996). El léxico y su didáctica: una propuesta metodológica. En Revista de Estudios de Adquisición de la Lengua Española. Universidad de Alcalá.

Leacock, Claudia; Chodorow, Martin. (1998). Combining Local Context and WordNet Similarity for Word Sense Identification. En: Fellbaum, Christiane (ed.) WordNet: and Electronic Lexical Database (pp. 265-274). Cambridge, Massachusetts: MIT Press.

Leoni de León, Antonio. (En prensa). De WordNet a la lexicografía interlingüística. Káñina.

Miller, George A., Beckwith, Richard; Fellbaum, Christiane; Gross, Derek; Miller, Katherine J. (1990). Introduction to WordNet: an on-line lexical database. International Journal of Lexicography 3 (4): 235-244. Recuperado el 24 de mayo del 2002, de ftp://ftp.cogsci.princeton.edu/pub/wordnet/5papers.pdf

Rojas, Marta. (2002). Léxico básico escolar costarricense. Diccionario de frecuencias. Informe de investigación. San José, Costa Rica: Universidad de Costa Rica.

. (2004). Desarrollo de la madurez léxica: Propuesta metodológica para el sexto año de la Educación General Básica. Informe de investigación. San José, Costa Rica: Universidad de Costa Rica.

Rojas, Marta; Sánchez, Víctor. Léxico Básico Escolar Costarricense. Investigación en proceso.

Vossen, Piek. (2001). Building a multilingual database with wordnets for several European languages. Recuperado el 25 de marzo del 2002, de http://www.hum.uva.nl/ ewn/index.html 


\section{Anexo A \\ Programa en Perl}

\section{Este programa convierte un archivo con valores separados por comas (CSV) en un documento XML:}

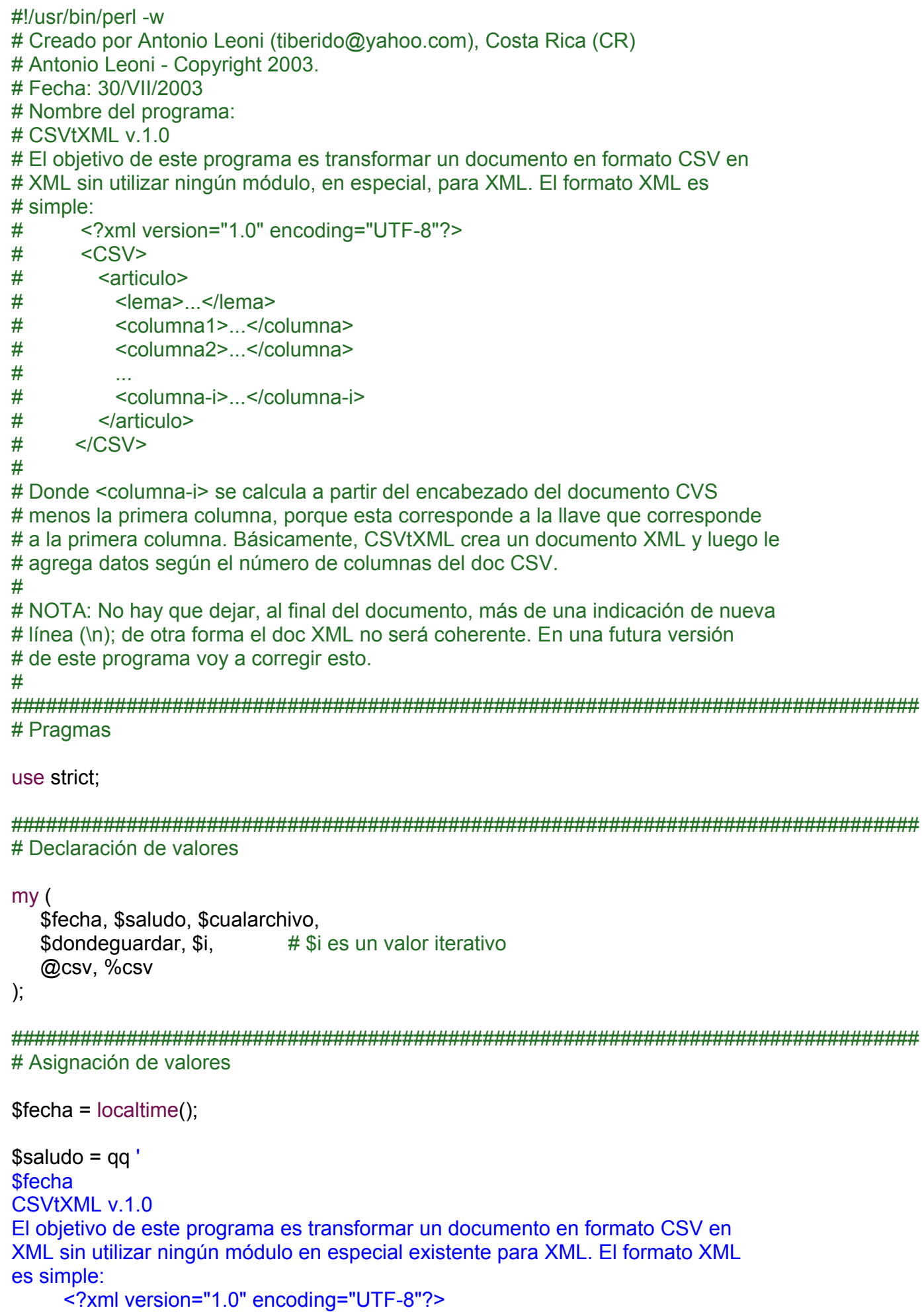




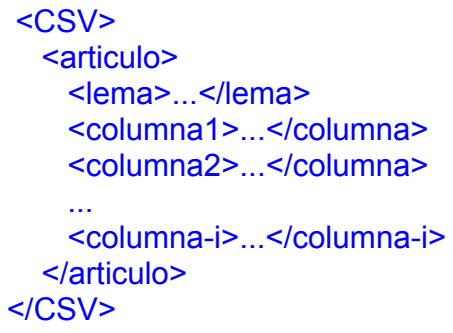

Donde <columna-i> se calcula a partir del encabezado del documento CVS menos la primera columna, porque esta corresponde a la llave que corresponde a la primera columna.

';

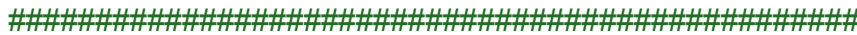

\#\# Inicio de subrutinas:

sub columnas \{

my ( \$unificado, @valoresenlascolumnas);

@valoresenlascolumnas = split(/\&/, \$_[0]),

chomp(@valoresenlascolumnas),

\$unificado = join ("In", @valoresenlascolumnas)

if defined(\$_[0]);

open(ADJUNTARCOLUMNA, ">> \$cualarchivo.xml") || die "No se puede abrir \$cualarchivo.xml: \$!"; \# Apertura del documento XML para adjuntar datos.

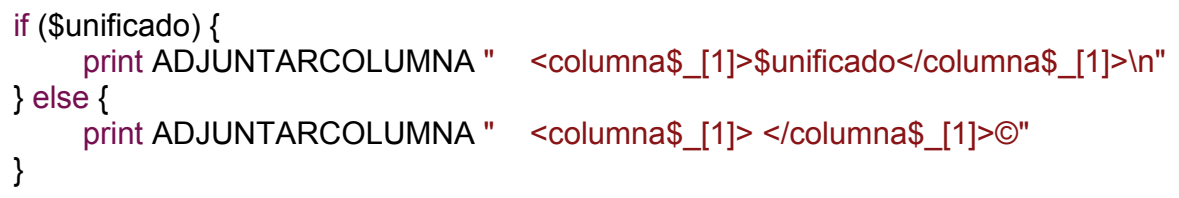


print CREARARCHIVO "<?xml version=|"1.0|" encoding=|"UTF-8|"?>In <CSV>।n"; close(CREARARCHIVO) || die "No se puede cerrar \$cualarchivo.xml: \$!";

open(LEERARCHIVO, "<\$cualarchivo") || die "No se puede abrir \$cualarchivo: \$!"; $\quad$ \# Apertura del archivo XML para adjuntar datos

print "Inicio de procedimientos...In";

while (<LEERARCHIVO>) \{ \# Mientras el archivo está abierto, \# Perl realiza las acciones siguientes

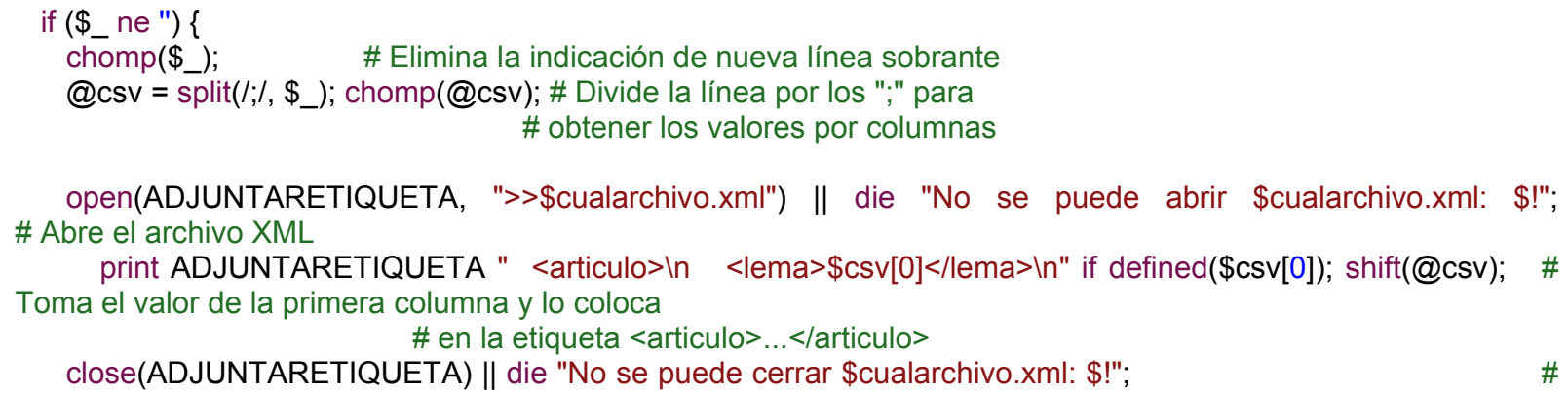




\section{Anexo B \\ Convertidor basado en XSLT}

Este programa transforma el documento resultante de la aplicación del programa en el Anexo A, en un documento XML próximo a AdjML:

$<$ ?xml version="1.0" encoding="UTF-8"?>

<xsl:stylesheet version="1.0" xmlns:xsl="http://www.w3.org/1999/XSL/Transform">

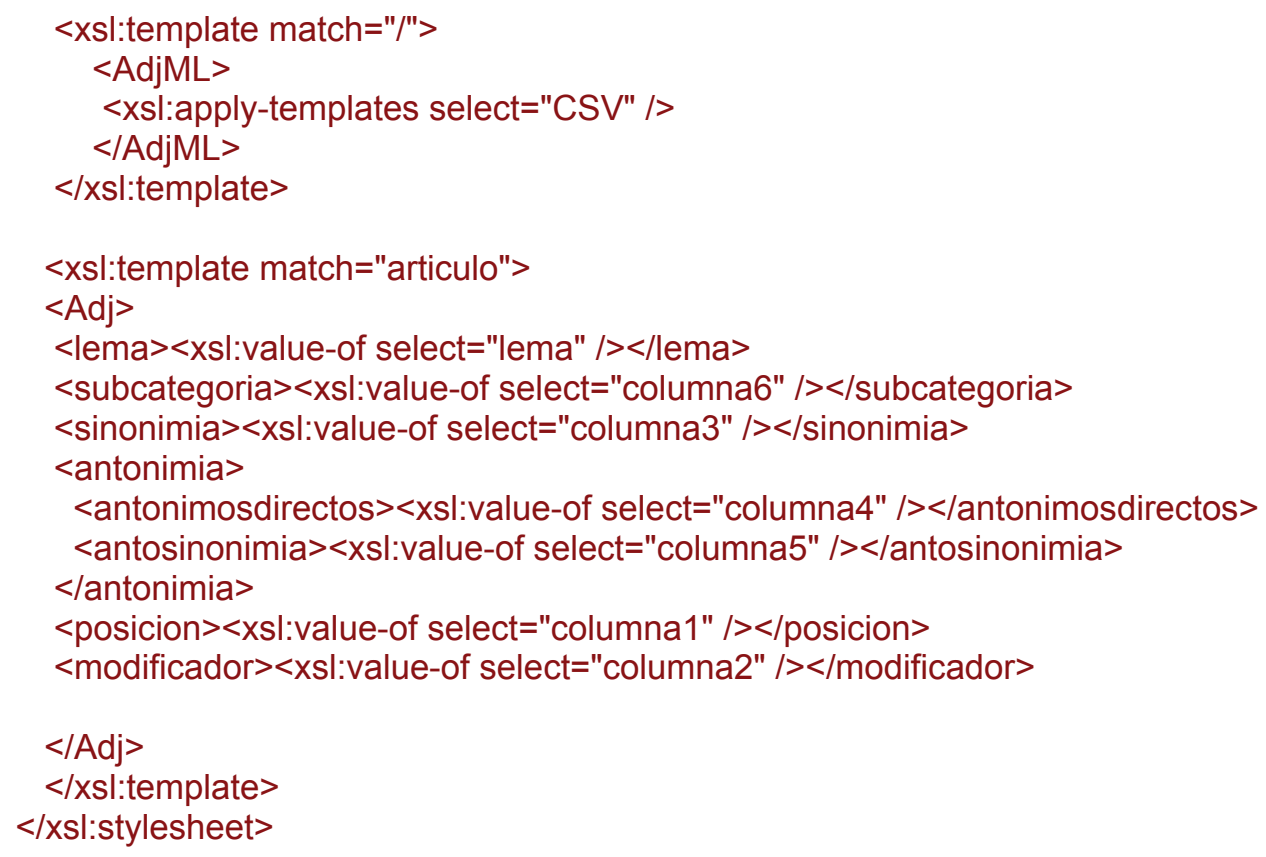




\section{Anexo C \\ Documento XML descriptor de adjetivos}

Presentación parcial del documento AdjML:

$<$ ?xml version="1.0"?>

$<$ AdjML $>$

$<$ Adj $>$

$<$ lema $>$ abierto(a)</lema $>$

$<$ subcategoria $>$ Descriptivo</subcategoria $>$

$<$ sinonimia $>$

*despejado *amplio *liberal *franco *directo

$</$ sinonimia $>$

$<$ antonimia $>$

<antonimosdirectos>

Cerrado

$<$ /antonimosdirectos>

$<$ antosinonimia>

Obtuso *estrecho * conservador *oculto *encubierto

$</ a n t o s i n o n i m i a>$

$</$ antonimia $>$

$<$ posicion>

libro abierto

$<$ posicion>

$<$ modificador $>$

Mi mente es abierta. El museo est\&\#xE1; abierto.

$<$ /modificador $>$

$</$ Adj $>$

$<$ Adj $>$

...

$<$ Adj $>$

$<$ AdjML $>$

$<$ AdjML $>$ 


\section{Anexo D \\ AdjML $\rightarrow$ HTML: antonimia}

El siguiente programa XSLT transforma el documento AdjML en un documento HTML que contiene los datos sobre la sinonimia y la antosinonimia:

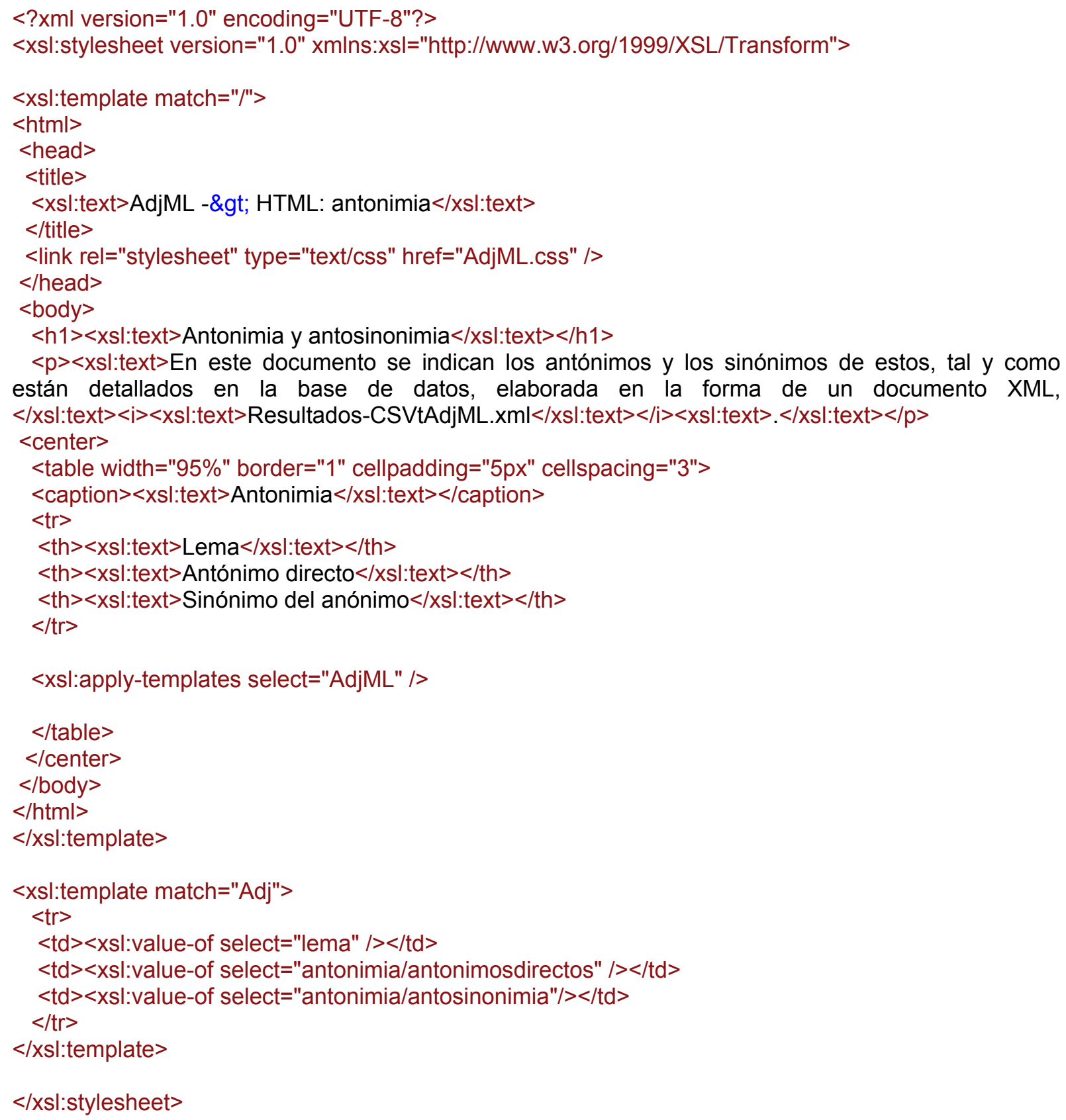




\section{Anexo E \\ Muestra del documento de valores separados por comas (CSV)}

similar; criterio similar; Ambos entornos son similares;

*semejante\&*parecido\&*paralelo\&*análogo\&*comparable\&*parangonable\&*emparentado\&*cercano; *disímil; " diferente $\&{ }^{*}$ distinto\&*disparejo\&*desigual\&opuesto\&*lejano;

vivo(a); Embajador bien vivo; El perro es vivo.\&; *astuto\&*sagaz\&*despierto\&*avispado\&*taimado\&*ladino\&*artero\&*jugado\&*malicioso; $\quad$ *ingenuo\&; ${ }^{*}$ confiado\& ${ }^{*}$ c $\ddagger$ ndido $\&{ }^{*}$ crŽdulo\&*incauto; Descriptivo

vivo(a); materia viva; El perro estł vivo.; ; " muerto; ; Descriptivo 


\section{Anexo F \\ Conversión de a LDA}

Este programa transforma el documento $\mathrm{XML}$ que resulta de la proyección de un documento CSV en XML, y lo convierte en el formato LDA:

$<$ ?xml version="1.0" encoding="UTF-8" ?>

= <xsl:stylesheet version="1.0" xmlns:xsl="http://www.w3.org/1999/XSL/Transform">

= <xsl:template match="l">

: $<$ AdjML $>$

$<$ xsl:apply-templates select="CSV" |>

$</$ AdjML $>$

$</ x$ sl:template $>$

= <xsl:template match="articulo">

$=<$ Adj $>$

= $\quad<$ lema postpuesto="\{columna1\}" antepuesto="\{columna2\}"

usopredpermanente="\{columna\}\}" usopredcontingente="\{columna4\}"

usopredsujoracional="\{columna5\}">

$<$ xsl:value-of select="lema" |>

$</$ lema $>$

$=<$ sinonimia $>$

$<$ xsl:value-of select="columna6" |>

$</$ sinonimia $>$

= <antonimia>

- <antonimosdirectos>

$<$ xsl:value-of select="columna7" |>

$</ a n t o n i m o s d i r e c t o s>$

= <antosinonimia>

$<$ xsl:value-of select="columna8" |>

$<$ /antosinonimia $>$

$<$ /antonimia $>$

$<$ Adj $\rangle$

$</ x$ sl:template $>$

$</ x s \mid$ stylesheet $>$ 


\section{Anexo G \\ Adjetivos compatible con estar}

Este programa extrae la información de compatibilidad con el verbo estar y crea un documento HTML con los resultados.

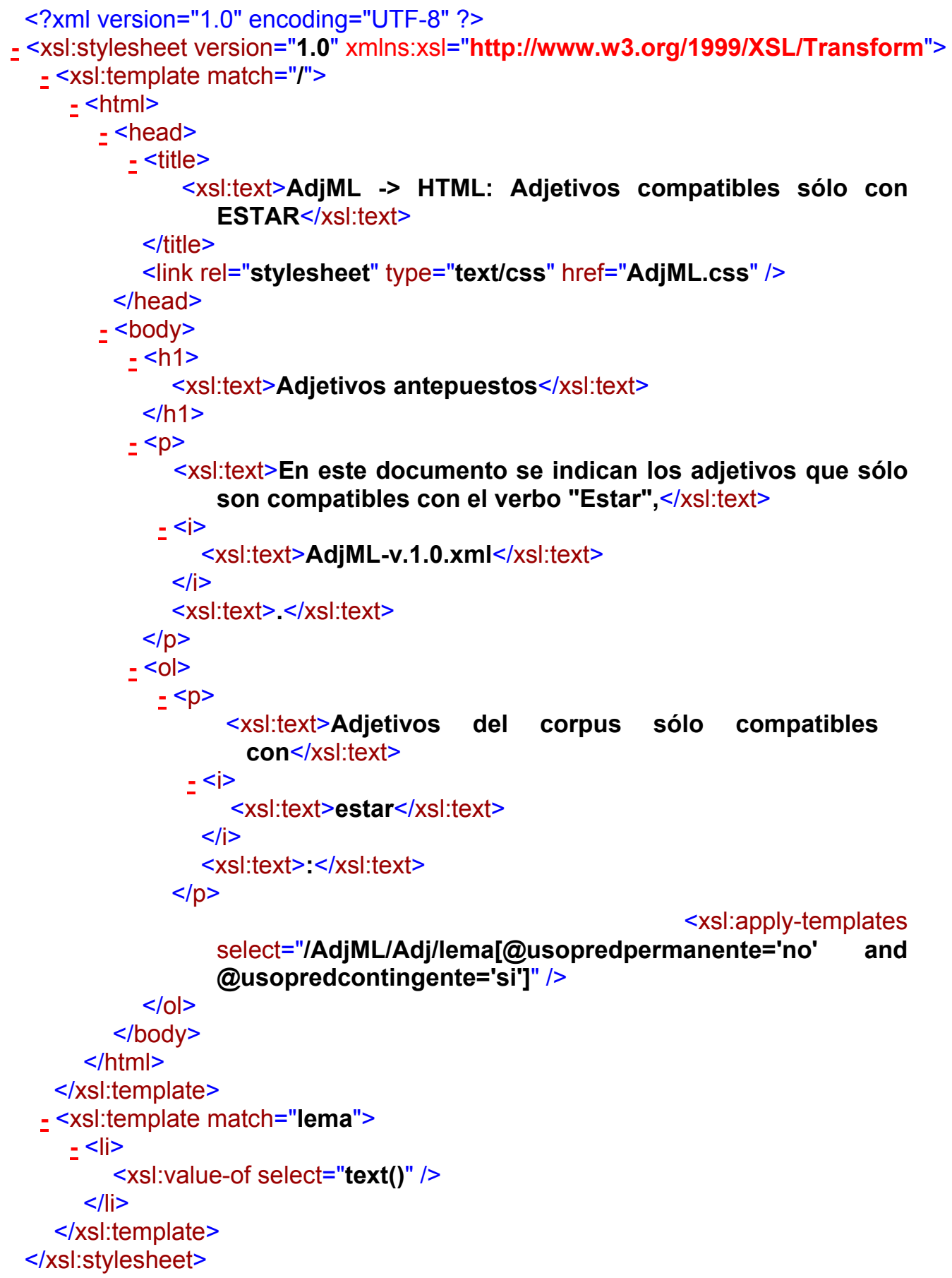




\section{Anexo H}

$<$ ?xml version="1.0" encoding="UTF-8" ?>

= <xsl:stylesheet version="1.0" xmlns:xsl="http://www.w3.org/1999/XSL/Transform">

= <xsl:template match="l">

$=<$ html $>$

$=<$ head $>$

$-<$ title>

$<$ xsl:text>AdjML $>>$ HTML: anteposición </xsl:text>

$</$ title $>$

$<$ link rel="stylesheet" type="text/css" href="AdjML.css" />

$</$ head $>$

$=<$ body $>$

$=<\mathrm{h} 1>$

$<x s \mid:$ text $>$ Adjetivos antepuestos $<|x s|: t e x t>$

$</$ h1 $>$

$=\langle\mathrm{p}\rangle$

$<x s l: t e x t>$ En este documento se indican los adjetivos que sólo

son antepuestos, extraídos a partir de </xsl:text $>$ $=<i>$

$<$ xsl:text $>$ LDA $</$ xsl:text $>$

$</ \mathrm{i}>$

$<$ xsl:text $>$.</xsl:text $>$

$</ p>$

$=<$ ol $>$

$=<\mathrm{p}>$

$<x s 1: t e x t>$ Lemas de adjetivos que se utilizan, en anteposición, según el corpus, son: $<|x s|: t e x t>$

$</ p>$

<xsl:apply-templates select="/AdjML/Adj/lema[@antepuesto='si' $</$ ol $>$ and @postpuesto='no']" />

$</$ body $>$

$</$ html $>$

$</ x$ sl:template $>$

= <xsl:template match="lema">

$=<\mid i>$

$<x$ l:value-of select="text()" |> $</ \mid i>$

$</ x s$ :template $>$

$</ x s \mid$ :stylesheet $>$

/xsl:template>

$</ x s l$ :stylesheet $>$ 